\title{
RSS-Based DoA Estimation in 802.11p Frequency Band Using ESPAR Antenna and PPCC-MCP Method
}

\author{
D. Duraj ${ }^{1}$, M. Tarkowski ${ }^{2}$, M. Rzymowski ${ }^{3}$, Member, IEEE, L. Kulas ${ }^{4}$, Senior Member, IEEE \\ and K. $\mathrm{Nyka}^{5}$, Member, IEEE \\ Department of Microwave and Antenna Engineering, Faculty of Electronics, Telecommunications and Informatics, \\ Gdansk University of Technology \\ Gdansk, Poland \\ ${ }^{1}$ damian.duraj@pg.edu.pl, ${ }^{2}$ michal.tarkowski@pg.edu.pl, ${ }^{3}$ mateusz.rzymowski@pg.edu.pl, ${ }^{4}$ lukasz.kulas@pg.edu.pl, \\ 5 nyx@eti.pg.edu.pl
}

\begin{abstract}
In this paper, the concept of direction of arrival (DoA) estimation using electronically steerable parasitic array radiator (ESPAR) antenna designed to operate in IEEE 802.11p vehicular communication standard has been investigated with respect to different possible elevation angles of a radio frequency (RF) signal impinging the antenna. To this end, two different possible sets of the 3D antenna radiation patterns have been used together with power-pattern cross-correlation (PPCC) algorithm and its extension that covers multiple calibration planes (PPCCMCP). Numerical simulations of the PPCC and PPCC-MCP angle estimation procedures at $5.89 \mathrm{GHz}$ have been performed to verify the overall DoA estimation accuracies in the horizontal plane for different, and unknown at the moment of estimation, elevation angles.

Index Terms-Electronically steerable parasitic array radiator (ESPAR) antenna, reconfigurable antenna, switched-beam antenna, DoA estimation, received signal strength (RSS), calibration, vehicle-to-everything
\end{abstract}

\section{INTRODUCTION}

Beam switching is one of the technique that is commonly used in wireless sensor network (WSN) [1]-[3] especially for localization purposes along with connectivity and safety improvement. One of the approaches to beam switching is electronically steerable parasitic array radiator (ESPAR) antenna [4] which uses one radiating element surrounded by multiple passive (parasitic) elements connected to variable loads. The modification of each element's load provides the possibility of altering antenna's properties. By placing passive elements on a ring and maintaining equal distances between them, it is possible to obtain symmetrical directional radiation patterns along with beam direction switching. Due to this fact, it is possible to estimate Direction-of-Arrival (DoA) of incoming signal with only one antenna output port [5].

This work was supported by SECREDAS project that has received funding from the Electronic Component Systems for European Leadership Joint Undertaking under grant agreement No 783119. This Joint Undertaking receives support from the European Union's Horizon 2020 research and innovation programme and Netherlands, Austria, Belgium, Czech Republic, Germany, Spain, Finland, France, Hungary, Italy, Poland, Portugal, Romania, Sweden, Tunisia, United Kingdom.
Many WSN applications utilize inexpensive, power efficient radio transceivers or microcontrollers, mainly armed with digital input/output (DIO) ports and limited number of integrated digital-to-analog converters (DAC) [3]. To address this limitation, ESPAR antenna designs that substitute complex variable reactance load with simple single-pole, double-throw (SPDT) switch that has only two possible impedance values so that it can be controlled with only two logic states were proposed [6]. Such concept can be adapted to perform DoA estimation with accuracy comparable to small arrays without necessity of using complex beamforming networks or separate antenna outputs for multiple independent radiating elements. With this simplified reconfiguration capability, it is possible to determine angle of incoming unknown signal with high precision, even using algorithms that depends only on received signal strength (RSS) captured by ESPAR antenna radiating element [7].

Interest in vehicle-to-everything (V2X) communication is increasing, especially in terms of $\mathrm{V} 2 \mathrm{X}$ systems functionality improvement with beamforming techniques, localization, incoming signals DoA estimation and also jamming mitigation. Due to this fact, a number of reconfigurable antenna designs were proposed [8]. It was proven that ESPAR antenna can be successfully adopted in V2X communication along with power pattern cross-correlation (PPCC) algorithm to obtain angle of arrival signal with precision under $7^{\circ}$ for multiple radiation pattern shapes, even with configurations that exhibit high halfpower beamwidth (HPBW) [9]. This gives the possibility not only to obtain acceptable angle estimation results but also for specific radiation patterns to maintain similar link quality between communicating objects in each direction.

In V2X systems maintaining the same elevation angle between objects is not possible without changing antennas orientations mechanically. Generally, the antenna in an infrastructure not always will be on the same height, but also different types of vehicle will try to communicate with system. This leads to constant change of arriving signal theta angle. In this paper we show that PPCC algorithm combined with 


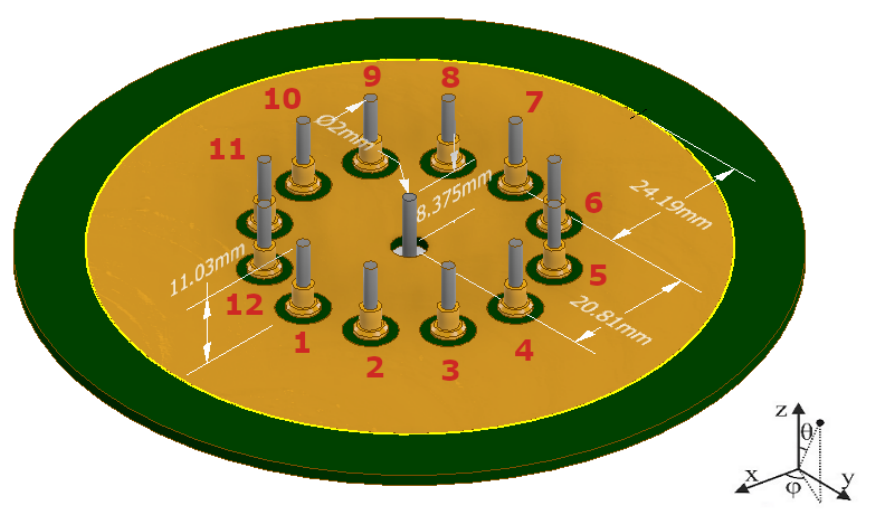

Fig. 1. 802.11p frequency band ESPAR antenna's design optimised for V2X application.

5.89 GHz ESPAR antenna can further improve DoA estimation results for $802.11 \mathrm{p}$ frequency band V2X applications with dynamically changing environment by involving multiple calibration planes (MCP) [10].

\section{ESPAR ANTENNA FOR V2X APPLICATIONS IN 802.11P FREQUENCY BAND}

The proposed antenna, shown in Fig. 1 and described in detail in [11], consists of a single active monopole fed by an SMA connector surrounded by twelve parasitic elements. The ground plane for radiating and passive elements and the layout for switching circuits are on the opposite layers of an inexpensive $1.55 \mathrm{~mm}$ thick FR4 substrate. Each parasitic element is connected to the switching circuit based on PE42424 SPDT switch that can change the load between open circuit or short circuit to the ground. In this design, the parasitic elements left open and shorted to the ground act as reflectors and directors, respectively. With this simple switching, circuit any microcontroller with only 12 digital output ports can be used to shape and rotate the antenna radiation pattern. It is possible to represent any ESPAR antenna configuration using steering vector which defines the combination of reflectors and directors: $V_{\max }^{n}=\left[v_{1}, v_{2}, \ldots, v_{\mathrm{s}} \ldots v_{12}\right]$, where $n$ represents radiation pattern number, while $v_{s}$ represents the setting of the s-th passive element which may take one of the two values: passive elements serves as director for $v_{s}=0$ and reflector for $v_{s}=1$.

In this article, only two configurations with their radiation patterns will be considered: five directors and seven reflectors (steering vector $V_{\max -D 5 R 7}^{1}$ ); six directors and six reflectors (steering vector $V_{\max -D 6 R 6}^{1}$ ). In Fig. 2 their radiation patterns were presented along with steering vectors and in Fig. 3 more detailed radiation pattern vertical and horizontal plane cuts were provided. Plots represent gain scaled in $\mathrm{dBi}$. In the proposed configurations, the antenna input impedance is well matched to $50 \mathrm{Ohm}$ preserving VSWR below 1.22 in the whole $802.11 \mathrm{p}$ frequency band. The difference between them are mainly in terms of maximum gain and half-power beamwidth (HPBW) in horizontal plane cut.

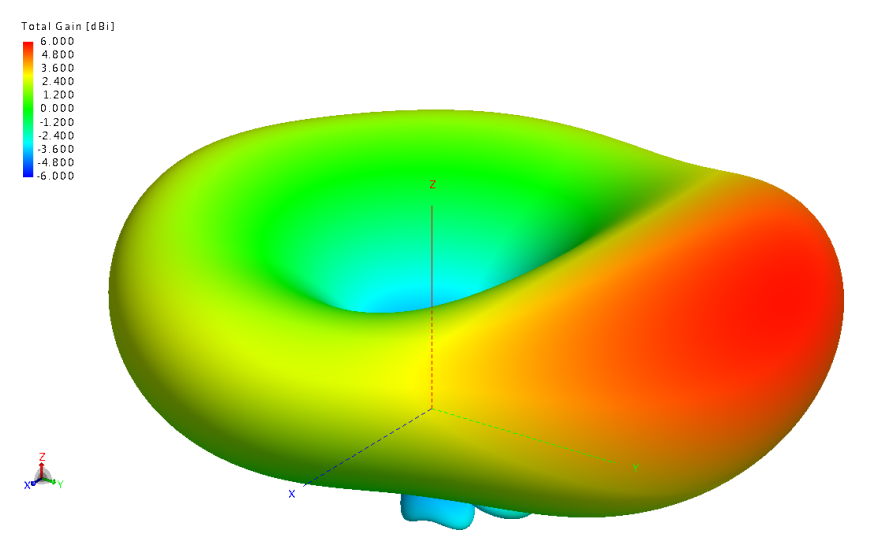

(a) $V_{\max -D 5 R 7}^{1}=[0,0,1,1,1,1,1,1,1,0,0,0]$

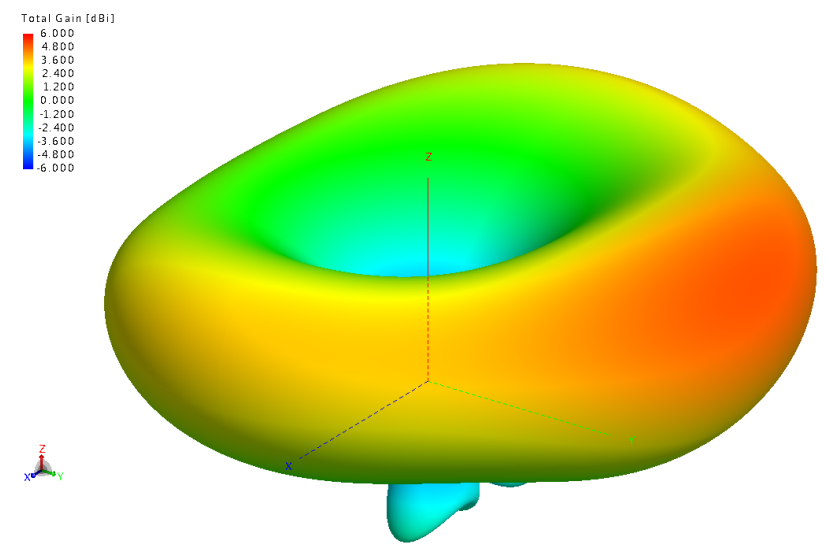

(b) $V_{\max -D 6 R 6}^{1}=[0,0,0,1,1,1,1,1,1,0,0,0]$

Fig. 2. $802.11 \mathrm{p}$ frequency band ESPAR antenna's radiation patterns with corresponding steering vectors.

Steering vectors can be associated with $\varphi_{\max }^{n}$ which defines the direction of the radiation pattern's maximum in horizontal plane cut. All results were prepared in a way that for each steering vector $V_{\max }^{1}$ the angle $\varphi_{\max }^{1}=0^{\circ}$ represents the maximum gain direction and by applying bit shift to this vector it is possible to rotate the radiation pattern by $30^{\circ}$ steps.

\section{RSS-BASED DOA ESTIMATION USING PPCC-MCP METHOD}

One of the methods which has proven its efficiency in DoA estimation is power pattern cross-correlation (PPCC) algorithm [12]. The concept is based on calculating correlation coefficients between received signal pattern $\left\{Y\left(V_{\max }^{1}\right), Y\left(V_{\max }^{2}\right), \ldots, Y\left(V_{\max }^{12}\right)\right\}$ formed by switching antenna's characteristic according to the steering vectors $\left\{V_{\max }^{1}, V_{\max }^{2}, \ldots, V_{\max }^{12}\right\}$ and its radiation patterns $\left\{\boldsymbol{p}^{1}, \boldsymbol{p}^{2}, \ldots, \boldsymbol{p}^{12}\right\}$ measured beforehand in the calibration phase with the angular step $\Delta \varphi$. These calculations result in the vector of correlation coefficients in the following form:

$$
\boldsymbol{g}=\frac{\sum_{n=1}^{12}\left(\boldsymbol{p}^{n} Y\left(V_{\max }^{n}\right)\right)}{\sqrt{\sum_{n=1}^{12}\left(\boldsymbol{p}^{n} \circ \boldsymbol{p}^{n}\right)} \sqrt{\sum_{n=1}^{12} Y\left(V_{\max }^{n}\right)^{2}}}
$$




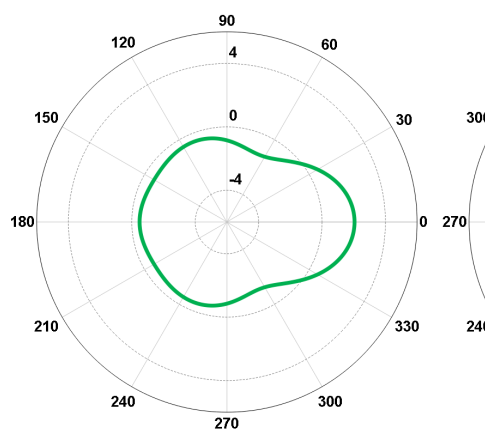

(a)

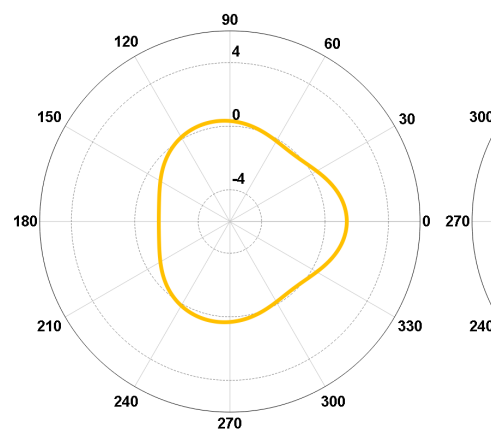

(c)

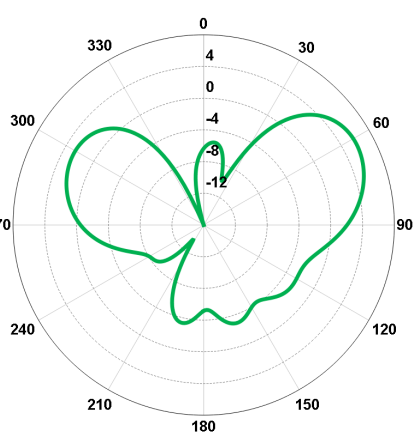

(b)

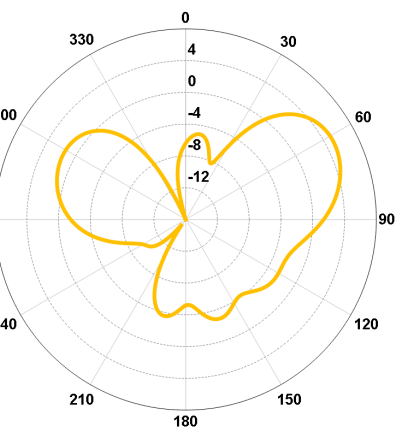

(d)

Fig. 3. $802.11 \mathrm{p}$ frequency band ESPAR antenna's radiation patterns horizontal (for $\theta=90^{\circ}$ ) and vertical (for $\varphi=0^{\circ}$ ) plane cuts for steering vectors $V_{\max -D 5 R 7}^{1}$ (respectively (a) and (b)) and $V_{\max -D 6 R 6}^{1}$ (respectively (c) and $(\mathrm{d}))$.

where the symbol ' $\circ$ ' stands for the Hadamard product, which is element-wise product of vectors. Vector $\boldsymbol{p}^{n}=\left[p_{1}^{n}, p_{2}^{n}, \ldots, p_{I}^{n}\right]^{T}$ contains discrete radiation pattern values corresponding to discrete horizontal angles $\varphi=$ $\left[\varphi_{1}, \varphi_{2}, \ldots, \varphi_{I}\right]^{T}$. The length $I$ depends on angular resolution of the calibration phase. Each element of vector $\boldsymbol{g}$ contains the correlation between received pattern and known characteristics, and thus the last step is to select the highest value and determine corresponding angle $\hat{\varphi}$ which is the final result of DoA estimation.

In its original form, PPCC used only one, horizontal calibration plane. To overcome this limitation, which emerges especially in IoT application with high variety of elevation angle, PPCC with multiple calibration planes (PPCC-MCP) was introduced [10]. It expands the calibration phase with additional $\mathbf{M}$ horizontal angles $\left\{\theta_{1}, \theta_{2}, \ldots, \theta_{M}\right\}$, so vector of radiation patterns can be written as:

$$
\boldsymbol{p}_{\theta}^{n}=\left[\left(\boldsymbol{p}_{\theta_{1}}^{n}\right)^{T},\left(\boldsymbol{p}_{\theta_{2}}^{n}\right)^{T}, \ldots,\left(\boldsymbol{p}_{\theta_{M}}^{n}\right)^{T}\right]^{T}
$$

Elements of $\boldsymbol{p}_{\theta}^{n}$ correspond to horizontal angles in expanded vector $\varphi_{\theta}$ :

$$
\boldsymbol{\varphi}_{\theta}=\left[\boldsymbol{\varphi}_{\theta_{1}}^{T}, \boldsymbol{\varphi}_{\theta_{2}}^{T}, \ldots, \varphi_{\theta_{M}}^{T}\right]^{T}, \bigwedge_{m} \boldsymbol{\varphi}_{\theta_{m}}=\varphi
$$

Thus, the length of the new coefficients vector equals $I * M$ and can be written as:

$$
\boldsymbol{g}_{\theta}=\frac{\sum_{n=1}^{12}\left(\boldsymbol{p}_{\theta}^{n} Y\left(V_{\max }^{n}\right)\right)}{\sqrt{\sum_{n=1}^{12}\left(\boldsymbol{p}_{\theta}^{n} \circ \boldsymbol{p}_{\theta}^{n}\right)} \sqrt{\sum_{n=1}^{12} Y\left(V_{\max }^{n}\right)^{2}}}
$$

Estimated angle $\hat{\varphi}$ is derived from $I * M$ elements of $\varphi_{\theta}$ which directly correspond to the highest value in $\boldsymbol{g}_{\theta}$.

\section{Numerical Simulations}

In order to verify, how the cumulative DoA estimation accuracy calculated using PPCC and PPCC-MCP algorithms is affected when impinging RF signal comes from different elevation angles, numerical simulations have been conducted. To this end, two considered sets of 3D antenna radiation patterns, which are associated with two sets of steering vectors $\left\{V_{\max -D 5 R 7}^{1}, V_{\max -D 5 R 7}^{2}, \ldots, V_{\max -D 5 R 7}^{12}\right\}$ and $\left\{V_{\max -D 6 R 6}^{1}, V_{\max -D 6 R 6}^{2}, \ldots, V_{\max -D 6 R 6}^{12}\right\}$, were generated for the proposed ESPAR antenna using computational electromagnetic program (FEKO) at $5.89 \mathrm{GHz}$, which is the center frequency of IEEE $802.11 \mathrm{p}$ standard, with $1^{\circ}$ angular step in horizontal and vertical directions. These patterns were used to generate calibration data for PPCC and PPCCMCP algorithms. For the PPCC case, calibration vectors $\left\{p^{1}, p^{2}, \ldots, p^{12}\right\}$ of length $I=360$ were created for each set of the steering vectors based on the horizontal cut (i.e. for thet $a=90^{\circ}$ ) of $3 \mathrm{D}$ radiation patterns only, while in the case of PPCC-MCP approach 9 horizontal angles $\left\{10^{\circ}, 20^{\circ}\right.$, $\left.30^{\circ}, 40^{\circ}, 50^{\circ}, 60^{\circ}, 70^{\circ}, 80^{\circ}, 90^{\circ}\right\}$ were used. Because the number of radiation pattern samples in horizontal direction $I$ $=360$, the resulting PPCC-MCP algorithm calibration vectors $\left\{\boldsymbol{p}_{\theta}^{1}, \boldsymbol{p}_{\theta}^{2}, \ldots, \boldsymbol{p}_{\theta}^{12}\right\}$ for each of the considered steering vectors set contain $360 * 9$ elements.

Numerical tests to evaluate PPCC and PPCC-MCP DoA estimation accuracy were performed in MATLAB. To this end, $10^{\circ}$ discrete angular step in both horizontal and elevation

TABLE I

Cumulative DoA Estimation ERRors For the Two CONSIDERED 802.11P ESPAR ANTENNA RADIATION PATTERNS CALCULATED USING PPCC AND PPCC-MCP ALGORITHMS (SEE TEXT FOR EXPLANATIONS)

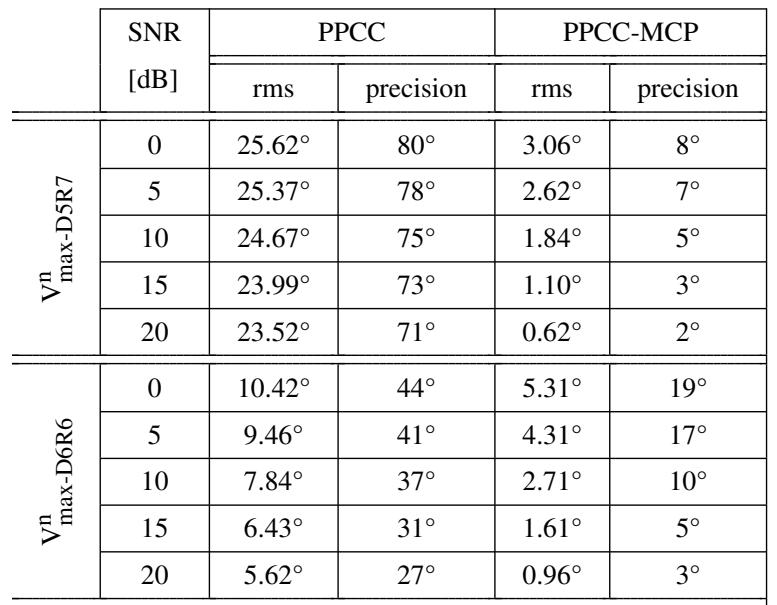


planes was used while the power of the test signal was set to $10 \mathrm{dBm}$ and, for every considered test direction, 10 snapshots of the received signal were captured. Additionally, additive white Gaussian noise was added to all recorded output power values to set a specific signal-to-noise ratio (SNR). In consequence, 36 test angles $\varphi_{t} \in\left\{0^{\circ}, 10^{\circ}, \ldots, 350^{\circ}\right\}$ in the horizontal direction and 9 test angles $\theta_{t} \in\left\{10^{\circ}, 20^{\circ}, \ldots, 90^{\circ}\right\}$ in the elevation plane were used giving 324 considered directions in total. Finally, twelve output power values, namely $Y\left(V_{\max -D 5 R 7}^{n}\right)$ and $Y\left(V_{\max -D 6 R 6}^{n}\right)$, required for DoA estimation process were recorded for every test direction for each of the two considered steering vectors sets.

PPCC algorithm DoA estimation results presented in Fig. 4 indicate that the algorithm performance do not change for elevation angles $\theta \geq 30^{\circ}$ when radiation patterns formed by the steering vectors $V_{\max -D 5 R 7}^{n}$ are used but deteriorates significantly for $\theta<20^{\circ}$. In the case of $V_{\max -D 6 R 6}^{n}$ steering vectors, DoA estimation accuracy stays at similar levels for $\theta \geq 40^{\circ}$ but the maximum DoA estimation error reaches much lower values. Additionally, the results gathered in Table I show that this effect is still present also for higher SNR values.

When PPCC-MCP algorithm is applied, the resulting DoA estimation errors are at acceptable levels for both considered ESPAR antenna radiation patterns, which can be seen in Fig. 5. The best results for all considered test signal angles were obtained when ESPAR antenna radiation patterns are generated using $V_{\max -D 5 R 7}^{n}$ steering vectors and the use of steering vectors $\mathrm{V}_{\text {max-D6R6 }}^{\mathrm{n}}$ in DoA estimation can result in higher errors. Moreover, the results gathered in Table I show that this effect can especially be pronounced in noisy environments. Nevertheless, ESPAR antenna radiation patterns generated using $V_{\max -D 6 R 6}^{n}$ steering vectors do not have strong maxima and deep minima in their horizontal cuts, which means that the connection quality is less likely to deteriorate during the radiation pattern rotation required to produce $\left\{Y\left(V_{\max }^{1}\right), Y\left(V_{\max }^{2}\right), \ldots, Y\left(V_{\max }^{12}\right)\right\}$ values for DoA estimation relying on PPCC or PPCC-MCP algorithms.

\section{CONCLUSIONS}

It has been shown that, by using PPCC-MCP algorithm, one can perform DoA estimation in the horizontal direction of RF signals impinging an ESPAR antenna designed for IEEE 802.11p frequency band with satisfying precision regardless of its elevation angle. Therefore, the presented approach raises low-cost and energy-efficient DoA estimation relying on ESPAR antennas designed for IEEE 802.11p frequency band to a more practical and applicable level, RF transmitters and receivers can be mounted on different heights. What is even more pronounced, such DoA estimation was also possible when antenna radiation patterns do not exhibit strong maxima or minima that can be used for maintaining better V2X connection during DoA estimation process. Because, in order to get closer to the real environment, the numerical

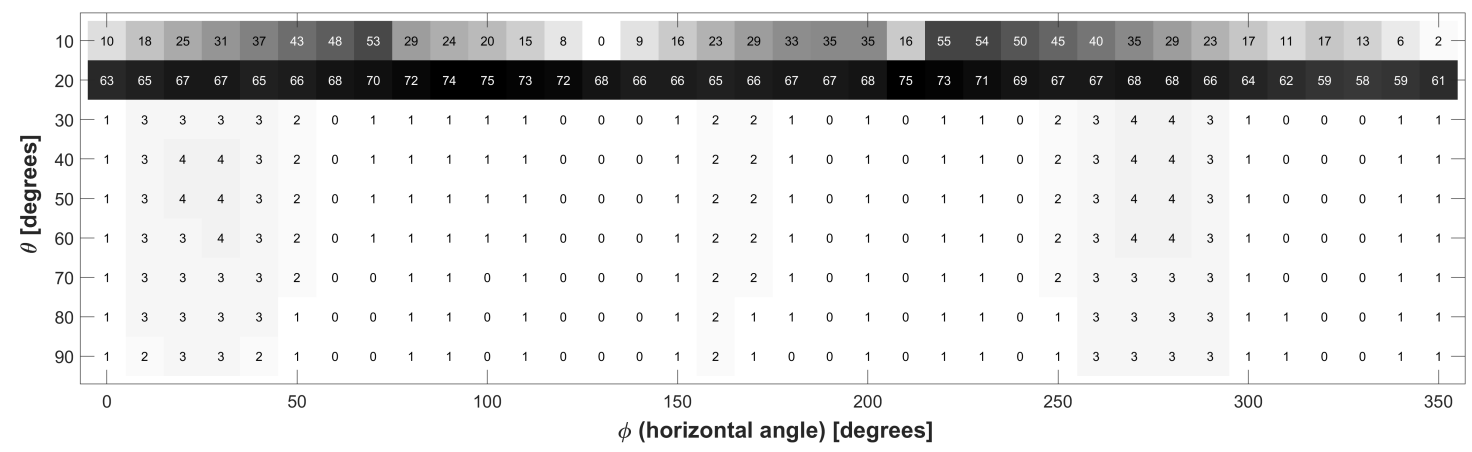

(a)

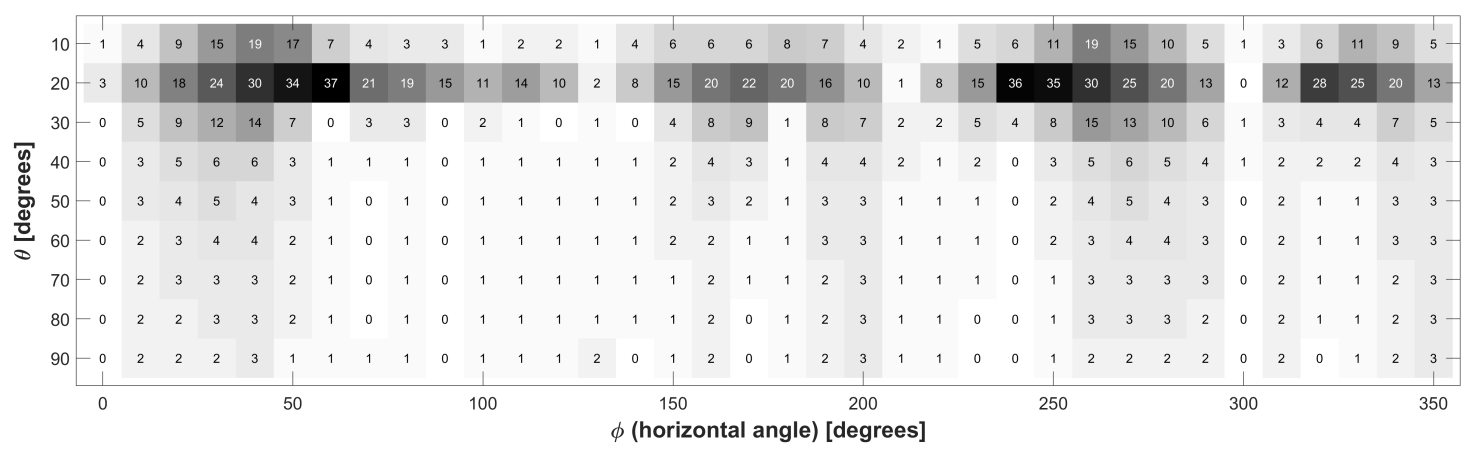

(b)

Fig. 4. Absolute values of DoA estimation errors calculated from numerical simulations using PPCC algorithm for incoming test signal angles changing in horizontal and elevation directions and $\mathrm{SNR}=10 \mathrm{~dB}$ when radiation patterns are created using $V_{\max -D 5 R 7}^{n}$ (a) and $V_{\max -D 6 R 6}^{n}$ (b) steering vectors (see text for explanations). 


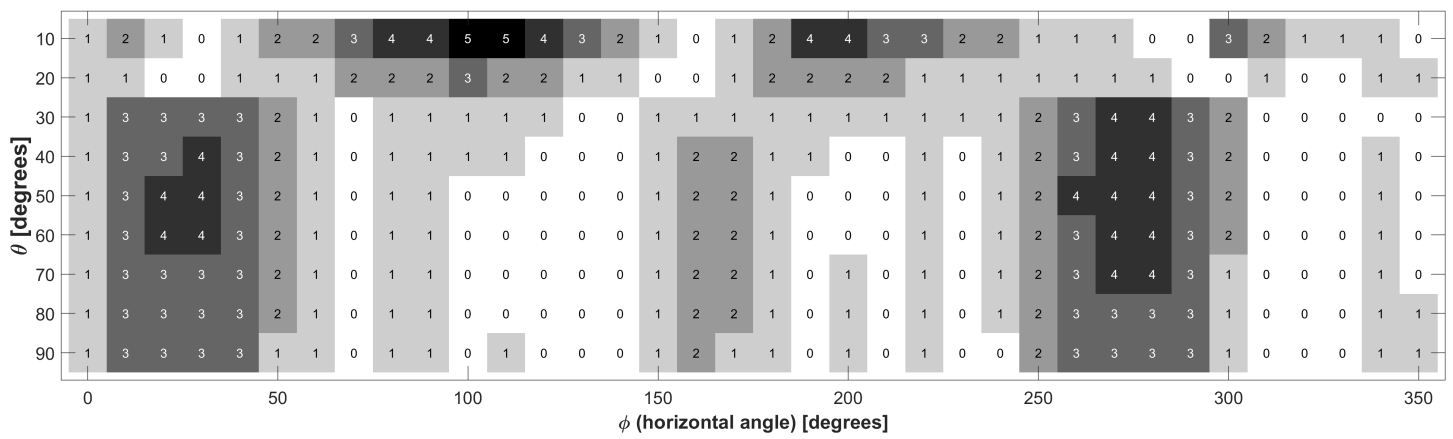

(a)

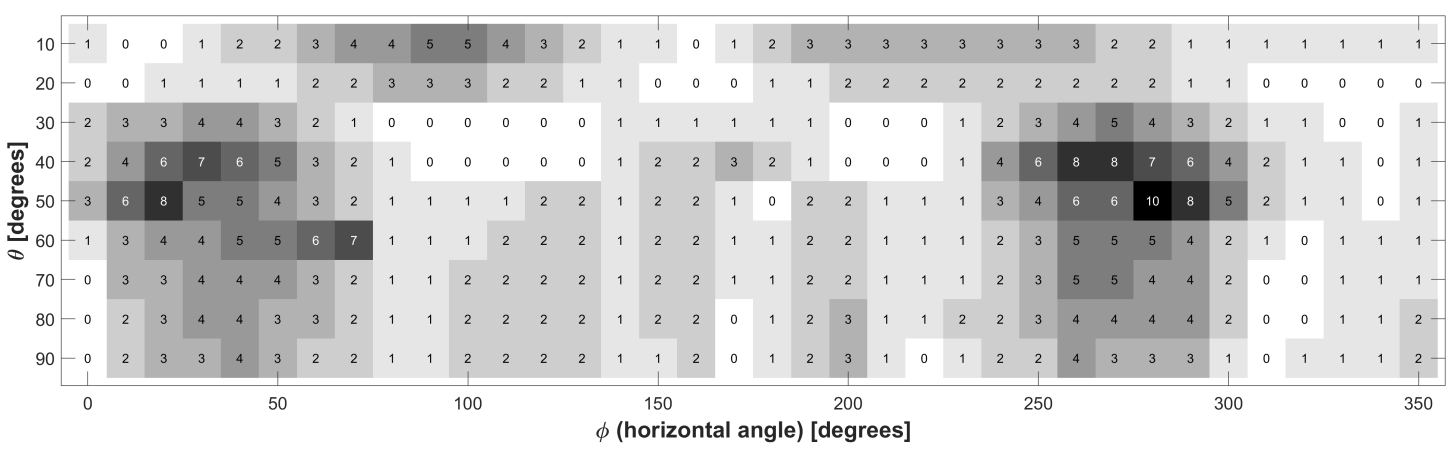

(b)

Fig. 5. Absolute values of DoA estimation errors calculated from numerical simulations using PPCC-MCP algorithm for incoming test signal angles changing in horizontal and elevation directions and $\mathrm{SNR}=10 \mathrm{~dB}$ when radiation patterns are created using $V_{\max }^{n}-D 5 R 7$ (a) and $V_{\max }^{n} D 6 R 6$ (b) steering vectors (see text for explanations).

simulations performed in the paper have been using different signal-to-noise ratios, it is expected that the promising results presented in this paper will bring this approach closer to the real tests in V2X environment, which are planned as the future follow-up work in this topic.

\section{ACKNOWLEDGMENT}

The authors would like to thank the Academic Computer Centre in Gdansk, Poland (TASK) were all the calculations were carried out.

\section{REFERENCES}

[1] L. Catarinucci, S. Guglielmi, R. Colella, and L. Tarricone, "Patternreconfigurable antennas and smart wake-up circuits to decrease power consumption in WSN nodes," IEEE Sensors Journal, vol. 14, no. 12, pp. 4323-4324, Dec 2014.

[2] E. Taillefer, A. Hirata, and T. Ohira, "Direction-of-arrival estimation using radiation power pattern with an ESPAR antenna," IEEE Transactions on Antennas and Propagation, vol. 53, no. 2, pp. 678-684, Feb 2005.

[3] M. Rzymowski, P. Woznica, and L. Kulas, "Single-anchor indoor localization using ESPAR antenna," IEEE Antennas and Wireless Propagation Letters, vol. 15, pp. 1183-1186, 2016.

[4] K. Gyoda and T. Ohira, "Design of electronically steerable passive array radiator (ESPAR) antennas," in IEEE Antennas and Propagation Society International Symposium. Transmitting Waves of Progress to the Next Millennium. 2000 Digest. Held in conjunction with: USNC/URSI National Radio Science Meeting (C, vol. 2, July 2000, pp. 922-925 vol.2.
[5] M. Pencole, E. Taillefer, T. Shimizu, and M. Taromaru, "Directionof-arrival estimation of highly correlated signals by applying forwardbackward averaging to the ESPRIT algorithm for 7-element ESPAR antenna," in The European Conference on Wireless Technology, 2005., Oct 2005, pp. 55-58.

[6] M. Rzymowski and L. Kulas, "Design, realization and measurements of enhanced performance $2.4 \mathrm{ghz}$ ESPAR antenna for localization in wireless sensor networks," in Eurocon 2013, July 2013, pp. 207-211.

[7] L. Kulas, "Simple 2-D direction-of-arrival estimation using an ESPAR antenna," IEEE Antennas and Wireless Propagation Letters, vol. 16, pp. 2513-2516, 2017.

[8] L. Marantis, K. Maliatsos, C. Oikonomopoulos-Zachos, D. K. Rongas, A. Paraskevopoulos, A. Aspreas, and A. G. Kanatas, "The pattern selection capability of a printed ESPAR antenna," in 2017 11th European Conference on Antennas and Propagation (EUCAP), March 2017, pp. 922-926.

[9] D. Duraj, M. Rzymowski, K. Nyka, and L. Kulas, "RSS-based DoA estimation using ESPAR antenna for V2X applications in 802.11p frequency band," (in press).

[10] M. Groth and L. Kulas, "Accurate PPCC-based DoA estimation using multiple calibration planes for WSN nodes equipped with ESPAR antennas," in 2018 15th European Radar Conference (EuRAD), Sep. 2018, pp. 545-548.

[11] D. Duraj, M. Rzymowski, K. Nyka, and L. Kulas, "ESPAR antenna for V2X applications in 802.11p frequency band," in 2019 13th European Conference on Antennas and Propagation (EuCAP), March 2019, pp. $1-4$.

[12] E. Taillefer, A. Hirata, and T. Ohira, "Direction-of-arrival estimation using radiation power pattern with an ESPAR antenna," IEEE Transactions on Antennas and Propagation, vol. 53, no. 2, pp. 678-684, Feb 2005. 